\title{
IMPROVED CAPACITIVE DETECTION METHOD FOR CORIOLIS MASS FLOW SENSORS ENABLING RANGE/SENSITIVITY TUNING
}

\author{
D. Alveringh ${ }^{1}$, J. Groenesteijn ${ }^{1}$, R.J. Wiegerink ${ }^{1}$, and J.C. Lötters ${ }^{1,2}$ \\ ${ }^{1}$ MESA+ Institute for Nanotechnology, University of Twente, Enschede, The Netherlands \\ ${ }^{2}$ Bronkhorst High-Tech BV, Ruurlo, The Netherlands
}

\begin{abstract}
We report on a novel capacitive detection principle for Coriolis mass flow sensors which allows for three times increased sensitivity. Capacitive Coriolis mass flow sensors are normally read out by two electrodes that measure the ratio between the actuation mode and the Coriolis mode, which is induced by the mass flow. This ratio results in a phase shift between the two electrodes. By adding two additional read out electrodes, the actuation mode signal is partially cancelled, allowing for higher sensitivity to the Coriolis mode, and thus larger phase shifts for the same mass flows. An analytical model is derived and correspond with the measurements. It is also proven that the range and sensitivity of the sensor can be tuned by changing the size of the additional readout electrodes. This readout method does not increase the readout time.
\end{abstract}

Keywords: Coriolis flow sensor, capacitive readout, phase detection, actuation mode cancellation.

\section{INTRODUCTION}

Coriolis mass flow sensors consist of a vibrating tube, which causes a fluid flowing inside the tube to experience Coriolis forces. As a result, a second vibration mode is excited with amplitude proportional to the mass flow. Coriolis forces are proportional to the mass and thus it is not straightforward to realize a miniaturized sensor.

A first attempt towards a micromachined Coriolis mass flow sensor was made by Enoksson et al. in 1997 [1]. Like most traditional Coriolis mass flow sensors they use an optical readout to detect the vibration modes. One year later they developed a capacitive readout for the sensor [2]. In 2001 another micromachined Coriolis mass flow sensor was presented by Smith et al., consisting of a highly doped silicon tube operating in vacuum [3]. In 2008, Haneveld et al. presented the first micro Coriolis mass flow sensor based on a silicon nitride tube with an extremely thin tube wall of $1.5 \mu \mathrm{m} \mathrm{[4].} \mathrm{This} \mathrm{sensor} \mathrm{did} \mathrm{not} \mathrm{require} \mathrm{opera-}$ tion in vacuum. A capacitive readout for this sensor was presented in 2009 [5]. For this readout combshaped electrodes were attached to the tube, see Figure 1. Comb shapes were used on the one hand because these were easy to implement in the fabrication process, and on the other hand to avoid squeeze film damping so that the sensor could still operate at atmospheric pressure.

With the readout capacitances $S_{1}$ and $S_{2}$ as indicated in Figure 1, the actuation mode results in differential and the detection mode results in common

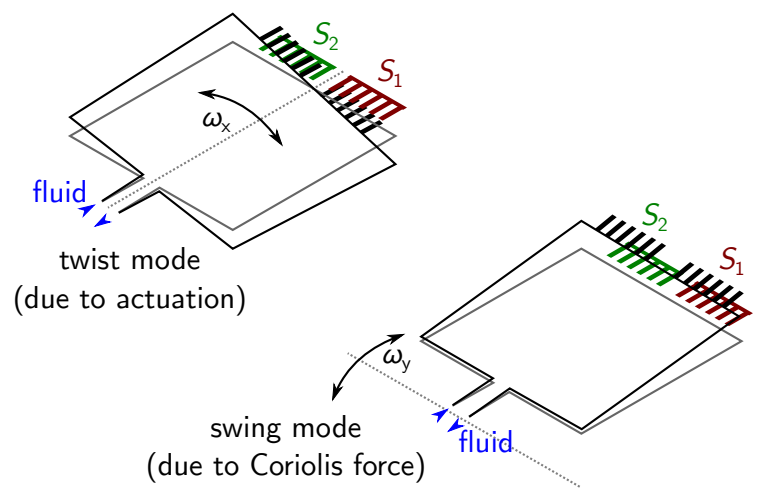

Figure 1: Operating principle of the Coriolis mass flow sensor [5]. The tube is actuated in twist mode $\omega_{\mathrm{X}}$ (top) which results in Coriolis forces. The Coriolis forces excite a swing motion $\omega_{\mathrm{y}}$ (bottom) with amplitude proportional to mass flow. Two combs at the tube produce signals $S_{1}$ and $S_{2}$ which are used to distinguish the two modes.

changes in capacitance. Since the Coriolis forces are proportional to the angular velocity of the tube, there is a $90^{\circ}$ phase shift between the actuation and detection mode. Without mass flow, the two signals from $S_{1}$ and $S_{2}$ have opposite phase. Mass flow results in additional phase shift proportional to the flow, as illustrated in Figure 2.

In many respects, the sensor presented in [4] has been steadily improved on various aspects $[6,7]$, however the capacitive readout has remained practically the same and the detection of the phase shift has become a limiting factor [8]. In this paper we present a method to increase the phase shift in order to improve the resolution as required by several applications, e.g. dosing of medications through intravenous therapy [9] and mass flow measurement in equipment for semiconductor industry.

\section{OPERATING PRINCIPLE}

The operating principle of the new capacitive readout is illustrated in Figure 2. Using the conventional readout the phase shift can be increased by moving the readout electrodes as close as possible towards the rotation axis, where the actuation mode amplitude is the smallest. However, in this way only limited improvement can be achieved without decreasing the size of the electrodes (which would increase the noise level). Therefore, in this paper we propose to add another pair of electrodes further away from the rotation axis, where the actuation mode amplitude is much larger. By scaling the size of these electrodes and connecting them in parallel to the readout electrode at the other side of the rotation axis it is possible 


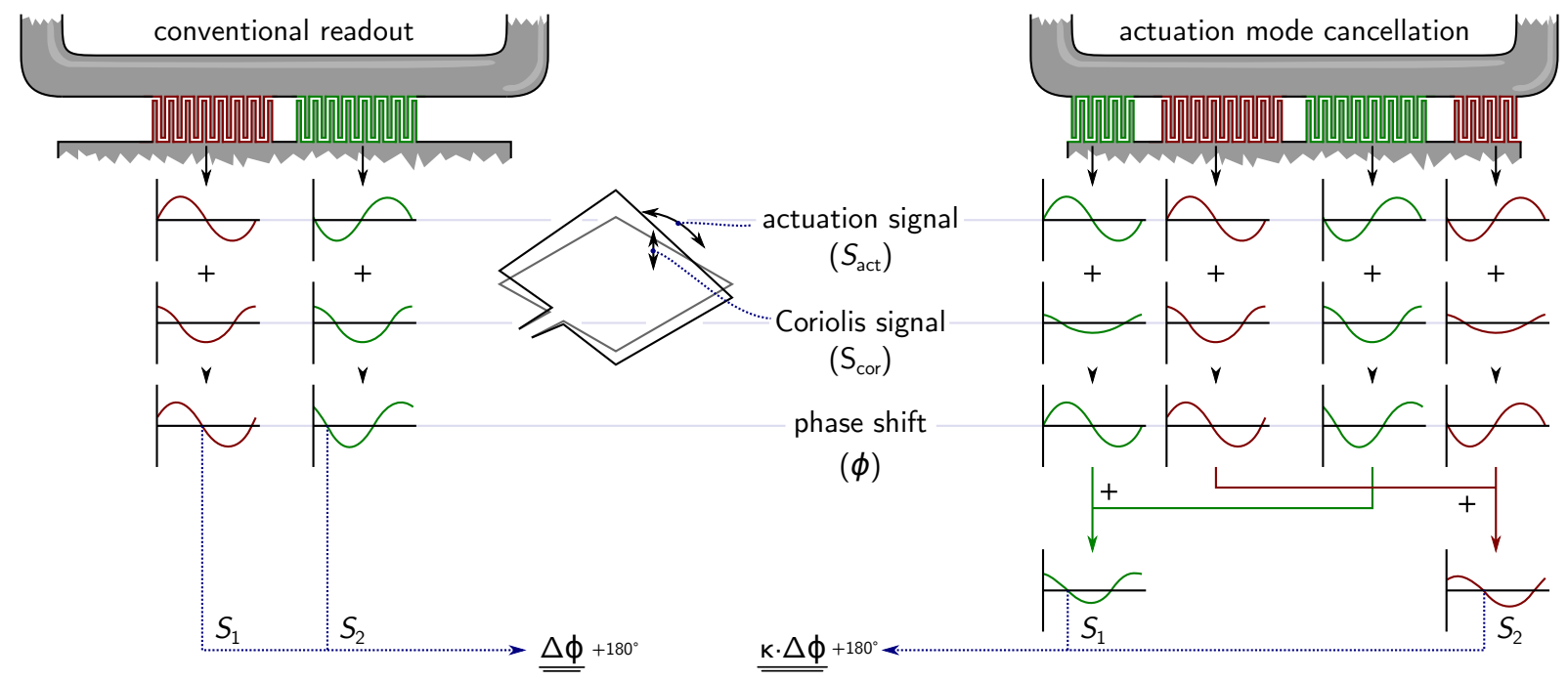

Figure 2: A conventional capacitive read out (left) provides a phase shift between the two output signals, however due to the small amplitude of the Coriolis motion the phase shift is small. The improved read out (right) uses two additional electrodes to cancel part of the twist mode; therefore, the phase shift is larger allowing for much higher sensitivity.

to cancel most of the actuation mode while keeping the same sensitivity for the detection mode. In this way a much larger phase shift is obtained at the same mass flow.

\section{Design}

Figure 3 shows the photomask design of the new sensor. The rectangular tube window is suspended at the center of one of the sides. The suspendend tube is in total approximately $14 \mathrm{~mm}$ long. Parallel tubes are used to reduce the overall pressure drop caused by the sensor.

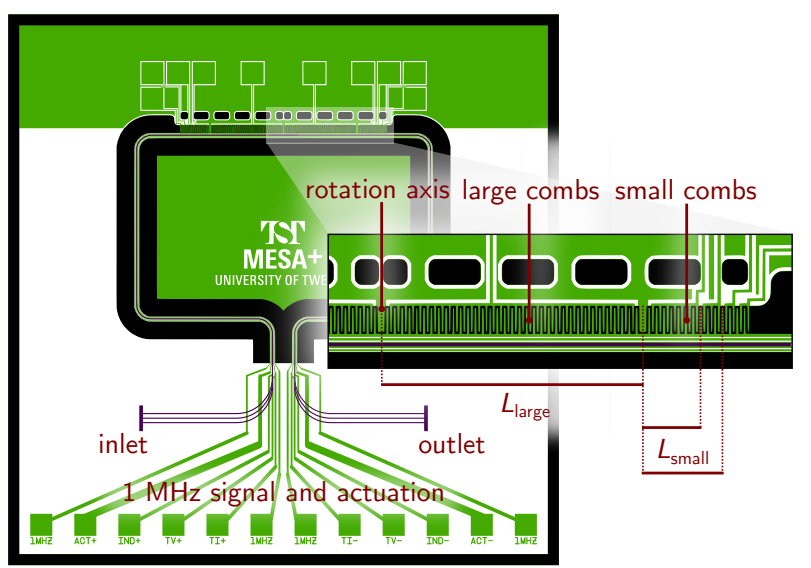

Figure 3: Photomask design of the new sensor, showing the new capacitive readout with large combs with width $L_{\text {large }}$ and additional small comb structures. The small combs have width $L_{\text {small }}$.

The readout electrodes are at the side opposite to the suspension. As before [5], comb-shaped electrodes are used. Due to residual stress the long silicon nitride tubes slightly bend upward, amounting to a deflection in the order of $10 \mu \mathrm{m}$ at the position of the electrodes. As a result, for small amplitudes the capacitance varies with the distance between the electrodes and thus with the motion of the tube.
The large combs have a width $L_{\text {large }}$ of approximately $950 \mu \mathrm{m}$ and the centers are located $475 \mu \mathrm{m}$ from the rotation axis (half of the width). The small combs have a width $L_{\text {small }}$ of approximately $200 \mu \mathrm{m}$ and are located $575 \mu \mathrm{m}$ from the rotation axis. The width of the small combs $L_{\text {small }}$ can be increased to $280 \mu \mathrm{m}$ with a distance of $615 \mu \mathrm{m}$ to the rotation axis. The actuation signal due to the twist mode is proportional to the width and the location of the combs, since the tube acts as a lever. The Coriolis signal due to the swing mode is only proportional to the width of the combs. This phenomenon makes discrimation between the two modes, and thus cancellation of the actuation mode signal, possible.

\section{AnAlysis}

Each of the two output signals $S_{1}$ and $S_{2}$ consists of an actuation mode component and a Coriolis mode component. The actuation mode component $S_{\text {act }}$ can be expressed as:

$$
S_{\text {act }}=A_{\text {act }} \cdot \sin (\omega t),
$$

with $A_{\text {act }}$ the amplitude, $\omega$ the frequency and $t$ the time. As mentioned above, the Coriolis component has a $90^{\circ}$ phase shift. Thus, it can be expressed as:

$$
S_{\text {cor }}=A_{\text {cor }} \cdot \cos (\omega t) \text {, }
$$

with $A_{\text {cor }}$ the amplitude of the Coriolis movement. The two capacitive output signals can now be written as:

$$
\begin{aligned}
& S_{1}=S_{\text {cor }}+S_{\text {act }}, \\
& S_{2}=S_{\text {cor }}-S_{\text {act }},
\end{aligned}
$$


or, by using Equations 1 and 2 and trigonometric identities:

$$
\begin{gathered}
S_{1}=A \cdot \sin (\omega t+\phi), \\
S_{2}=-A \cdot \sin (\omega t-\phi),
\end{gathered}
$$

with:

$$
\begin{gathered}
A=\sqrt{A_{\text {act }}^{2}+A_{\text {cor }}^{2}}, \\
\Delta \phi=\arctan \left(\frac{A_{\text {cor }}}{A_{\text {act }}}\right) .
\end{gathered}
$$

with $\Delta \Phi$ the phase shift between $S_{1}$ and $S_{2}$. For small phase shifts, $\tan (\Delta \phi) \approx \Delta \phi$; the phase shift is proportional to the ratio of the Coriolis mode amplitude and actuation mode amplitude. From this, it follows that the phase shift can be increased (factor $\kappa$ ) by reducing the actuation signal. This can be achieved by subtracting the signal of the small combs from the signal of the large combs.

$$
\kappa \cdot \Delta \phi=\frac{A_{\text {cor }}}{A_{\text {act }}}=\frac{A_{\text {cor }}}{A_{\text {large }}-A_{\text {small }}} .
$$

The factor $\kappa$ is equal to the ratio between the conventional readout and the readout with cancellation:

$$
\kappa=\frac{A_{\text {large }}}{A_{\text {large }}-A_{\text {small }}},
$$

and can be estimated using the geometric definitions in Figure 3. As mentioned before, the amplitude of the large combs $A_{\text {large }}$ is proportional to the product of the width of the comb $\left(L_{\text {large }}\right)$ and the distance between the center of the comb and the rotation axis $\left(L_{\text {large }} / 2\right)$ :

$$
A_{\text {large }} \propto L_{\text {large }} \cdot \frac{L_{\text {large }}}{2} .
$$

The amplitude of the small combs $A_{\text {small }}$ is in a similar way proportional to the geometry:

$$
A_{\text {small }} \propto L_{\text {small }} \cdot\left(L_{\text {large }}+\frac{L_{\text {small }}}{2}\right) .
$$

With the approximated values $L_{\text {large }}=950 \mu \mathrm{m}$ and $L_{\text {small }}=200 \mu \mathrm{m} \vee 280 \mu \mathrm{m}$, the following cancellation factors are estimated using Equations 10, 11 and 12:

- No cancellation: $\kappa=1$;

- $L_{\text {small }}=200 \mu \mathrm{m}: \kappa=1.9$;

- $L_{\text {small }}=280 \mu \mathrm{m}: \kappa=3.1$.

\section{FABRICATION}

Fabrication is done using surface channel technology based on [10] and is illustrated in Figure 4. The process starts with a silicon-on-insulator wafer with a handle layer of $400 \mu \mathrm{m}$, a buried oxide layer of $5 \mu \mathrm{m}$ and a device layer of $50 \mu \mathrm{m}$ (Figure 4a). Approximately $1 \mu \mathrm{m}$ of silicon nitride is deposited using low pressure chemical vapor deposition (Figure
$4 \mathrm{~b})$. Then, using deep reactive ion etching, the inlets are realized in the bottom of the wafer (Figure 4c). A pattern of slits is etched in the silicon nitride and the silicon below is etched using isotropic dry etching (Figures $4 \mathrm{~d}$ and $4 \mathrm{e}$ ) until a diameter of approximately $40 \mu \mathrm{m}$. The buried oxide layer at the inlets is etched using HF (Figure 4f). Another deposition step of silicon nitride is done (Figure $4 \mathrm{~g}$ ), which seals the slits and forms the tube (with a wall of approximately $1 \mu \mathrm{m})$. Finally, the tubes are released by isotropic dry etching (Figures $4 \mathrm{~h}, 4 \mathrm{i}$ and $4 \mathrm{j}$ ). Due to residual stress in the silicon nitride, the suspended tube bends upward.

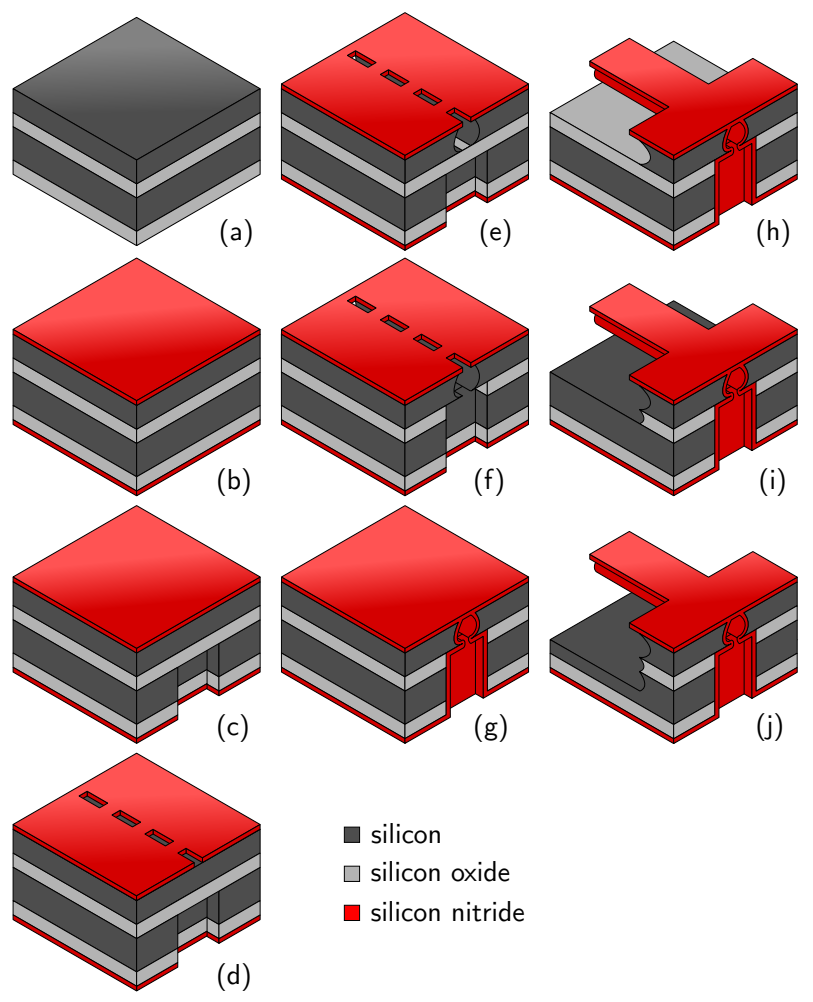

Figure 4: Fabrication process of the micro Coriolis sensor.

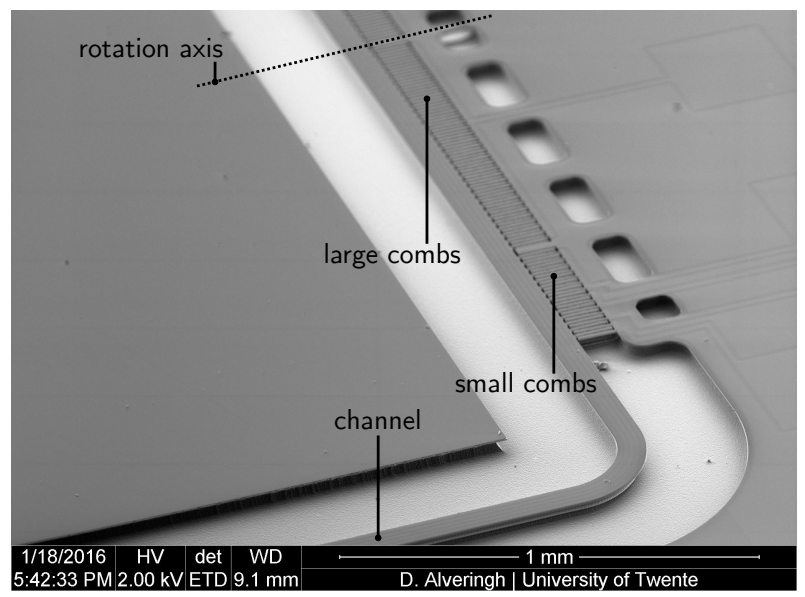

Figure 5: SEM close up of the tube with the large and small combstructures at one side of the tube. 
A scanning electron microscopy (SEM) close-up is shown in Figure 5. The chip is glued on a printed circuit board (PCB). The inlet and outlet are at the bottom of the chip and directly connected to holes in the PCB. A 3D-printed fluid connector is glued at the other side of the PCB. A photograph of the assembled device is shown in Figure 6.

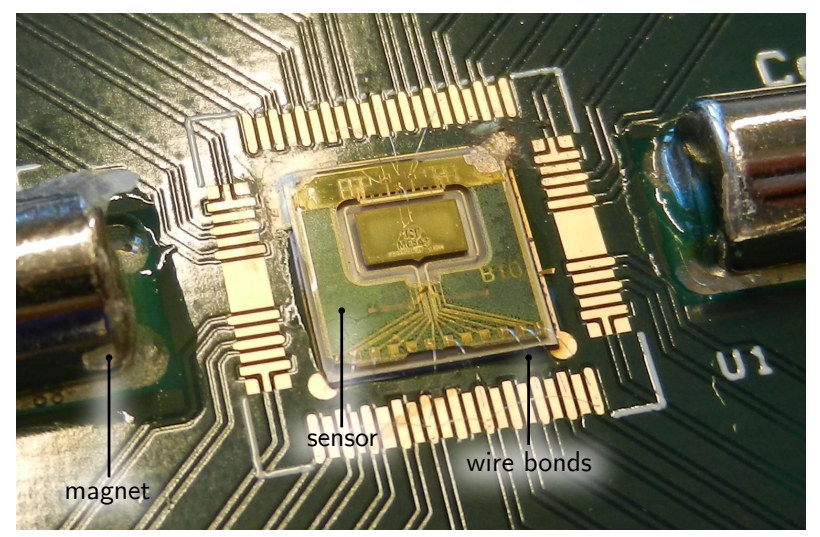

Figure 6: Assembled sensor on PCB with magnets and electronic interface. The fluid connector is at the other side of the PCB.

\section{Characterization}

The fabricated devices were characterized by applying a flow and measuring the phase shift between the two output signals for three situations: no actuation mode cancellation, actuation mode cancellation with $\kappa=1.9$ and actuation mode cancellation with $\kappa=3.1$.

A well-defined mass flow is applied using a commercially available mass flow controller (Bronkhorst M12p) in combination with a pressurized tank filled with deionized water. The mass flow controller is placed after the device under test to prevent it from contaminating the micro channels, see Figure 7.

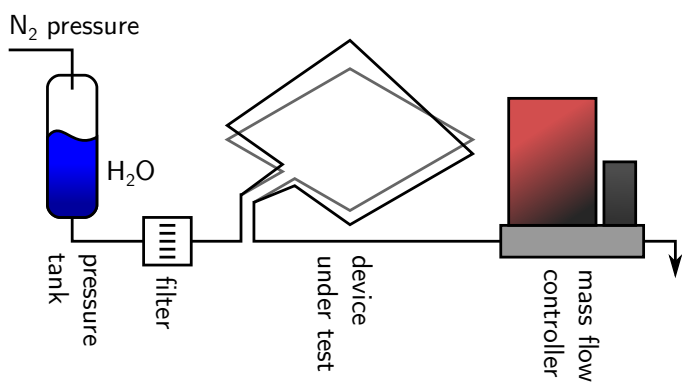

Figure 7: Fluidic measurement setup.

Figure 8 shows a block schematic of the interface electronics used for capacitive readout. A $1 \mathrm{MHz}$ carrier signal is applied to the comb structures on the moving tube. The fixed comb structures are kept at virtual ground by using two charge amplifiers. The resulting amplitude modulated signals are demodulated using analog multipliers (SA602) and low-pass filters, giving analog voltages proportional to the capacitances. Two lock-in amplifiers (Stanford SR830) are used to detect the phase shift between these signals.

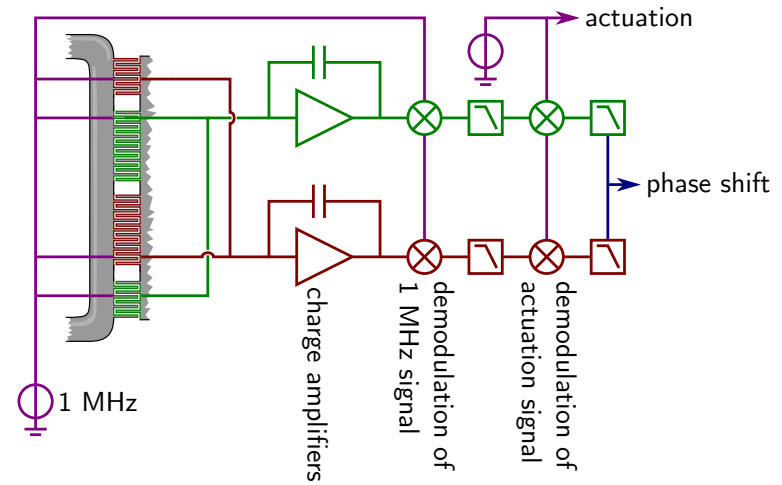

Figure 8: Block schematic of the electronic measurement setup.

Figure 9 shows measurement results for mass flow of water from $0 \mathrm{~g} \mathrm{~h}^{-1}$ up to $10 \mathrm{~g} \mathrm{~h}^{-1}$, without and with actuation mode cancellation. Arctan-fits are applied through the measurements. The theoretical cancellation $\mathcal{K}$ of 1.9 and 3.1 result in approximately the same measured increases in phase shifts, as is described in Equation 9.

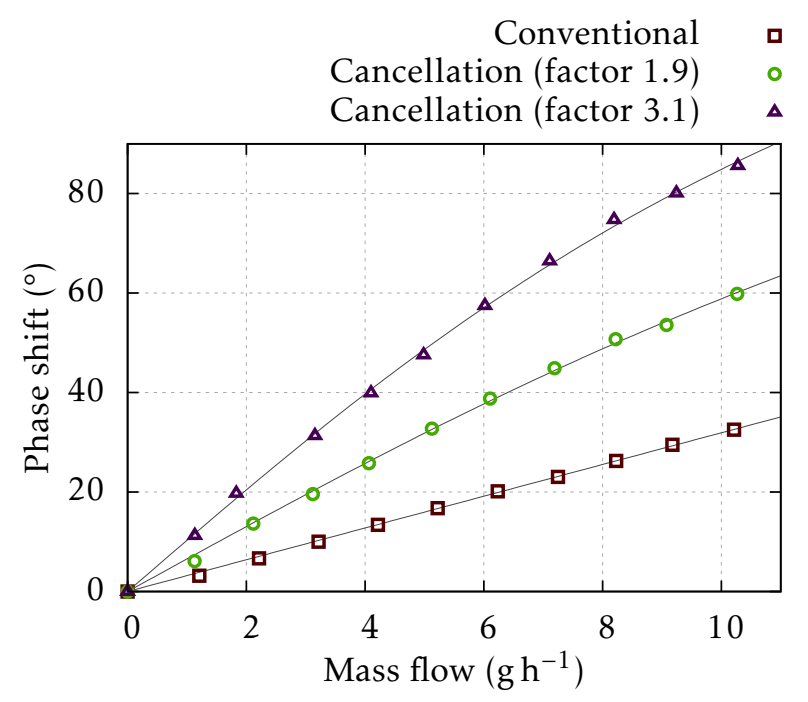

Figure 9: Conventional readout and improved readout measurement results using actuation mode cancellation. The phase shift increases a factor 1.9 or 3.1 dependent on the amount of cancellation.

\section{Discussion}

The amplitudes of the signals fed to the readout electronics become lower with higher cancellation as is described by Equation 7 . Increasing the actuation amplitude of the tube solves this. However, this is limited, since the combs should not cross eachother; the electrodes at the moving tube should always be above the electrodes of the stator.

Future research will focus on preventing the crossing of combs and finding the optimum between actuation mode cancellation and signal amplitude. E.g. by placing the combs for cancellation at the other (anchor) side of the Coriolis sensor.

We will also investigate the possibility of feeding a $1 \mathrm{MHz}$ signal with a different amplitude to the small 
combs. In this way, cancellation can be linearly tuned by the oscillator.

\section{CONCLUSION}

A micro Coriolis mass flow sensor with actuation mode cancellation is designed and fabricated. This method allows for higher phase shifts for the same mass flows and increases the sensitivity while using phase detection electronics with the same resolution. From the first measurements, it can be concluded that the concept can increase the sensitivity by a factor three and that it can be described by an analytical model. This novel readout method does not introduce a longer readout time and can be tuned for specific ranges and sensitivities without redesign or refabrication.

\section{ACKNOWLEDGEMENTS}

The authors gratefully acknowledge the financial support by the Eurostars Programme through the TIPICAL project (E!8264) and the technical help by R.G.P. Sanders and K. Ma.

\section{REFERENCES}

[1] P. Enoksson, G. Stemme, E. Stemme, A silicon resonant sensor structure for coriolis mass-flow measurements, Journal of Microelectromechanical Systems 6 (2) (1997) 119-125.

[2] T. Corman, P. Enoksson, G. Stemme, Lowpressure-encapsulated resonant structures with integrated electrodes for electrostatic excitation and capacitive detection, Sensors and Actuators A: Physical 66 (1) (1998) 160-166.

[3] R. Smith, D. R. Sparks, D. Riley, N. Najafi, A mems-based coriolis mass flow sensor for industrial applications, Industrial Electronics, IEEE Transactions on 56 (4) (2009) 1066-1071.

[4] J. Haneveld, T. S. J. Lammerink, M. A. Dijkstra, H. Droogendijk, M. J. de Boer, R. J. Wiegerink, Highly sensitive micro coriolis mass flow sensor, in: 21st IEEE International Conference on Micro Electro Mechanical Systems, MEMS 2008, 2008, pp. 920-923.

[5] J. Haneveld, T. S. J. Lammerink, M. J. de Boer, R. J. Wiegerink, Micro coriolis mass flow sensor with integrated capacitive readout, in: $22 \mathrm{nd}$ IEEE International Conference on Micro Electro Mechanical Systems, MEMS 2009, IEEE, 2009, pp. 463-466.

[6] J. Groenesteijn, T. S. J. Lammerink, R. J. Wiegerink, J. Haneveld, J. C. Lötters, Optimization of a micro coriolis mass flow sensor, Procedia Engineering 25 (2011) 783-786.
[7] J. Groenesteijn, H. Droogendijk, R. J. Wiegerink, T. S. J. Lammerink, J. C. Lötters, R. G. P. Sanders, G. J. M. Krijnen, Parametric amplification in a micro coriolis mass flow sensor: reduction of power dissipation without loss of sensitivity, in: IEEE SENSORS 2013, IEEE, 2013, pp. 1-4.

[8] J. Groenesteijn, Microfluidic platform for coriolis-based sensor and actuator systems, Ph.D. thesis, University of Twente, Enschede (January 2016).

[9] D. Alveringh, R. J. Wiegerink, J. C. Lötters, Towards system-level modeling and characterization of components for intravenous therapy, Proceedings of the 2nd International Conference on MicroFluidic Handling Systems, MFHS 2014 (2014) 106-109.

[10] J. Haneveld, T. S. J. Lammerink, M. J. De Boer, R. G. P. Sanders, A. Mehendale, J. C. Lötters, M. Dijkstra, R. J. Wiegerink, Modeling, design, fabrication and characterization of a micro coriolis mass flow sensor, Journal of Micromechanics and Microengineering 20 (12) (2010) 125001. 